\title{
Simultaneous diagnosis of tuberculous and non- tuberculous mycobacterial diseases: Time for a better patient management
}

\author{
Yeya DS Sarro' ${ }^{1}$, Bourahima Kone ${ }^{1}$, Bassirou Diarra ${ }^{1}$, Alisha Kumar ${ }^{2}$, Ousmane Kodio ${ }^{1}$, Djeneba B Fofana ${ }^{1}$, Chad J Achenbach ${ }^{2}$, Abdoul H \\ Beavogui ${ }^{3}$, Moussa Seydi ${ }^{4}$, Jane L Holl ${ }^{2}$, Babafemi Taiwo ${ }^{2}$, Souleymane Diallo ${ }^{1}$, Seydou Doumbia ${ }^{1}$, Robert L Murphy' ${ }^{2}$ Sally M McFall ${ }^{2}$ and \\ Mamoudou Maiga ${ }^{1,2 *}$
}

${ }^{1}$ University of Sciences, Techniques and Technologies of Bamako, Bamako, Mali

${ }^{2}$ Northwestern University, Chicago, Illinois, USA

${ }^{3}$ Universite Gamal Abdel Nasser de Conakry (UGANC), Conakry, Guinea

${ }^{4}$ Universite Cheikh Anta Diop, Dakar, Senegal

\begin{abstract}
Tuberculosis (TB) is the deadliest infectious disease in the world which disproportionately affects low-and-middle-income countries (LMICs) where diagnostic resources and treatment options are limited. The incidence of pulmonary non-tuberculous mycobacteria (NTM) disease is also rapidly increasing in these regions traditionally dominated by TB infections. This poses significant diagnostic and treatment challenges, since these two diseases are often indistinguishable clinically or by sputum smear microscopy (SSM), the most commonly used TB diagnostic tool in LMICs. Consequently, NTM-infected patients usually receive unnecessary TB treatment for months. TB patients with NTM co-infections may also be treated incorrectly due to inaccurate SSM and Xpert ${ }^{\mathrm{TM}} \mathrm{MTB} / \mathrm{RIF}$ ( $M$. tuberculosis/rifampin) results. These issues complicate the management of patients and contribute to the worsening of the current TB and NTM epidemiological features including development of drug resistant strains. It is therefore critical to develop improved diagnostic tools to accurately distinguish these two different pathogens that have many similar clinical and epidemiological features but have different treatment regimens. In this review, we will discuss limitations with current diagnostic tools and the need to develop novel techniques that can accurately and simultaneously diagnose TB and NTM disease.
\end{abstract}

\begin{abstract}
Abbreviations: TB: Tuberculosis; NTM: Non-Tuberculous Mycobacteria; SSM: Sputum Smear Microscopy; XDR: Extensively Drug Resistant; LMICs: Low-and-Middle-Income Countries; HIC: High-Income Countries; WHO: World Health Organization; MAC: Mycobacterium Avium Complex; MABC: Mycobacterium Abscessus complex; AFB: Acid-Fast Bacillus; HPLC: High-Performance Liquid Chromatography
\end{abstract}

\section{Introduction}

Tuberculosis (TB) is the most common deadly infectious disease worldwide and remains a major public health threat especially in the developing world. The incidence of the disease is 275 per 100,000 population in the African region, while in American and European regions, it is 27 and 36 per 100,000 population, respectively [1]. In parallel, the incidence and prevalence of nontuberculous mycobacteria (NTM) are also increasing both in low-and-middle-income countries (LMICs) and high-income countries (HICs) [2]. The prevalence of NTM in North America and Australia ranges from 3.2 to 9.8 per 100,000 population whereas in Africa, its prevalence is between 4 to $15 \%$ of "TB suspects" [3-11]. NTM include all mycobacteria other than Mycobacterium tuberculosis complex (MTBC, TB causative agent) and $M$. leprae (leprosy causative agent). The NTMs are composed of more than 150 species some of which are capable of causing disease in humans (e.g., M. avium, M. simiae, M. palustre, M. fortuitum, M. kansasii, M. kumamotonense, M. kubicae and M. morokaense). All these mycobacterial species appear positive on TB regular sputum smear microscopy (SSM), which makes the assay less specific for TB. This SSM assay that is more than 100 years old, is still the most widely available TB diagnostic tool, particularly in LMICs. SSM also has limited sensitivity and misdiagnoses half of tuberculosis cases. The World Health Organization (WHO) now recommends Xpert ${ }^{\mathrm{Tx}}$ MTB/ RIF (M. tuberculosis/rifampin) assay and other molecular tools to address some of the inaccuracies associated with SSM. Xpert ${ }^{\mathrm{tm}}$ MTB/ RIF is more sensitive and more specific than SSM, but less sensitive than sputum culture (the current gold standard). Xpert ${ }^{\mathrm{m}}$ MTB/RIF also cannot adequately differentiate TB and NTM infections. Sputum culture is available mostly in HICs, but it is time consuming (10 to 42 days) and requires very expensive infrastructures that are not accessible in LIMCs where it is most needed [12,13]. It has to be noted that only $57 \%$ of TB cases reported in 2014 were bacteriologically-confirmed while the remaining cases were treated empirically [1]. These cases include TB Xpert-negatives, children with paucibacillary TB and people living with HIV $[14,15]$. The objective of this particular review is to

*Correspondence to: Mamoudou Maiga, Center for Innovation in Global Health Technologies, Northwestern University, USA, Tel: +1-847-467-2560, E-mail: mamoudou.maiga@northwestern.edu

Key words: tuberculosis, non-tuberculous mycobacteria, epidemiology and diagnostic

Received: November 16, 2018; Accepted: November 27, 2018; Published: November 30, 2018 
inform clinicians, biologists and technology developers on the current epidemiological features of TB and pulmonary NTM disease, and the need for developing new and more appropriate diagnostic tools.

\section{Tuberculosis and NTM overlap in epidemiology and clinical presentations}

NTM infections were recognized to be very prevalent in HICs and were believed to be less frequent in LMICs. This is likely because of its overlap with TB infections in LMIC regions and the lack of affordable diagnostic tests. NTM disease has now been well-documented to be not only extremely common in LMICs but to be an important management challenge for TB patients themselves. Moreover, its prevalence is continuously increasing in both HICs and LMICs [16-18]. We and others have shown that the failure to accurately detect NTM in TB endemic countries may lead to the misdiagnosis of "chronic pulmonary TB-like cases" that have regular TB symptoms including cough, fever, weight loss, hemoptysis, malaise and fatigue. This misdiagnosis of NTM cases may result in unnecessary lengthy, expensive, and potentially toxic TB treatments $7,17,19[7,16,18]$. Our team recently reported the first three cases of extensively drug resistant TB (XDR-TB) in Mali. This was secondary to the incorrect diagnosis of a MTBC and NTM co-infection, and the use of SSM as the follow-up assay which led to drug resistance in the first index case [19]. This first case of XDRTB had persistent positive SSM during treatment, making it difficult to discern which TB treatment regimen to follow. SSM was at that time the only diagnostic test available through the Mali National TB Program. This patient's treatment was changed from a first to secondline TB regimen. However, after reanalysis of the patient's original stored samples, the patient was determined to have had a MTBC and NTM co-infection explaining the persistence of SSM positivity while receiving an effective TB treatment [19]. The patient subsequently developed XDR-TB, and in turn, infected two close contacts (confirmed by spoligotyping patterns) [19]. Overall, we found in Mali that $12 \%$ of TB suspected and treated cases were in fact infected by NTM, and of these, $73 \%$ were Mycobacterium avium complex (MAC) [7]. Similar data has been reported from Burkina Faso, Nigeria, Kenya, and many other LMICs where NTM is predominant $[18,20,21]$. It is not clear why NTM diseases have been recently increasing worldwide, particularly cases of pulmonary MAC [16]. Environmental changes, HIV infection, aging, immunosuppression treatments, malignancy and autoimmune diseases are known associated factors that may explain this recent increase $[16,22,23]$.

\section{Current diagnostic tools for TB and NTM infections}

The current diagnostic strategy, if pertinent assays are available, is to test sequentially for TB and then for NTM. This takes time and resources, leading to new transmissions, disease progression and development of drug resistance. The most important diagnostic methods currently available include:

Sputum smear microscopy with acid-fast bacillus (AFB) staining is the primary method for diagnosis of pulmonary tuberculosis in LMICs [24]. It is simple, rapid, and inexpensive but lacks sensitivity with a limit of detection of $10,000 \mathrm{CFU} / \mathrm{mL}$ of sputum and misses up to half of $\mathrm{TB}$ cases $[14,25,26]$. Its specificity is also poor, especially in areas where MTBC and NTM coexist [16].

Sputum culture is the gold standard and requires only $10 \mathrm{CFU} / \mathrm{mL}$ to be positive [27]. However, it must be performed in a high containment laboratory and requires expensive equipment, often unavailable in LMICs. In addition, it takes nearly 10 days for liquid medium-based cultures to be positive (example: MGIT ${ }^{\text {rox }}$ for Mycobacteria Growth Indicator Tube for example) and does not differentiate MTBC from NTM. The solid medium-based cultures have somewhat better specificity than the liquid medium, but require 3-6 weeks to grow [12]. Morphologic characteristics on solid media can help differentiate MTBC from NTM species but due to its lack of accuracy needs further confirmation with molecular assays such as AccuProbe making this strategy even more expensive [7].

Molecular methods are now more preferred than biochemical tests and high-performance liquid chromatography (HPLC) for mycobacterial species' identification [28]. The most utilized include: direct probe hybridization assays, line probe assays (LPA) and Xpert ${ }^{\mathrm{m}}$ MTB/RIF.

The most used direct probe hybridization assay is AccuProbeGenprobe. AccuProbe Culture Identification system (AccuProbe; Hologic, Inc., San Diego, CA, USA) is a US FDA-approved commercial test for identification of MBTC, MAC, M. gordonae, and M. kansasii from growth in solid or liquid culture media [29,30]. AccuProbe molecular assay has excellent specificity in differentiating MTBC from NTM, but has limited sensitivity requiring use of a "pre-culture" to yield enough nucleic acids [31]. Thus, AccuProbe is complementary to sputum culture. Accuprobe, however, does not overcome the challenges, expense and laboratory requirements needed for cultures and the need to test simultaneously for both TB and NTM limiting its usefulness in LMICs. Line probe assays (LPAs) are probe-based with complementarity with the reverse hybridization of PCR products. This rapid assay method allows for simultaneous identification of multiple mycobacterial species with good sensitivity $[29,32]$. However, the test has limited specificity leading to frequent false positives and false negatives, due to unsuccessful hybridization from heterogeneity in the probe-binding region [33]. Many commercial LPAs are available including INNO-LiPA Mycobacteria (Fujirebio Europe, Ghent, Belgium); GenoType Mycobacterium (Hain Lifescience, Nehren, Germany); Speed-oligo Mycobacteria (Vircell, Granada, Spain); AdvanSure Mycobacteria GenoBlot Assay (LG Chem Inc., Seoul, South Korea); and REBA Myco-ID (YD Diagnostics, Yongin, South Korea) [29].

Xpert $^{\mathrm{tm}}$ MTB/RIF Assay is a TB rapid automated nucleic acid amplification assay (Cepheid, USA) endorsed by the WHO in 2010 which enables diagnosis of TB and simultaneous assessing rifampicin resistance. The use of the assay has proven to be feasible in LMICs despite its advanced technology. However, it has limited performance for smear negatives and cannot detect NTM species [18,25]. With a detection limit of $131 \mathrm{CFU} / \mathrm{mL}$, Xpert ${ }^{\mathrm{ma}}$ is significantly less sensitive than sputum culture (at $10 \mathrm{CFU} / \mathrm{mL}$ ). A negative Xpert result needs to repeat and confirmed by sputum culture which increases the cost $[34,35]$. Sputum smear negativity is frequent with HIV co-infected individuals and children in LMICs and impacts the clinical utility of Xpert $^{\text {ma }}$ MTB/RIF Assay in these regions. Recently Cepheid released a new improved version of the assay called Xpert ${ }^{\mathrm{su}}$ MTB/RIF Ultra (Xpert Ultra) to overcome some of the limitations associated with the original version [36]. Xpert Ultra has slightly better sensitivity compared to the original version approximating $91 \%$ for smear positives but has a lower specificity [36]. Xpert Ultra is not as accurate as sputum culture, with a sensitivity of only $88 \%$ for all TB culture positive cases, only $63 \%$ for smear negatives, and $91 \%$ for smear positives [36]. Therefore, there is still an unmet need for improved-molecular diagnostic assays for TB, including the ability to simultaneously diagnose NTM infections. These assays need to have higher levels of accuracy and be easily accessible to both HICs and LMICs. Our team at the Center for Innovation in 
Global Health Technologies (CIGHT) at Northwestern University has developed a molecular assay that is as sensitive as sputum culture (XtracTB Assay), which we believe will revolutionize molecular diagnostics for TB and greatly improve patient management $[14,15]$. By adding NTM panels to this new TB molecular assay, we hope to overcome many of the issues described above. Such highly sensitive TB/NTM assays will have significant positive impacts on TB and NTM disease management worldwide.

\section{NTM disease is not routinely diagnosed despite avail- ability of treatments}

Globally, MAC is the most common cause of pulmonary NTM disease in patients with and without HIV co-infection [16,37]. Unlike many other NTM diseases, MAC infection is treatable [7,22], if an accurate diagnosis can be made. Treatments and outcomes differ depending on the NTM species [22]. SSM is generally the only direct bacteriological test available [12]. Patients are usually suspected of having NTM disease only after evidence of non-existence of TB or failure of lengthy TB treatment regimens $[23,38]$. A recent large, international multi-center study with 30 participant countries worldwide identified MAC as the most common NTM species, followed by M. gordonae, M. xenopi, and M. kansasii [39,40]. However, Mycobacterium abscessus complex (MABC) and M. fortuitum were the most common rapid mycobacterial growers [39,40]. Therapeutic decisions are challenging and often empiric because pulmonary NTM diseases' symptoms are similar for all pulmonary mycobacterial diseases including TB [39]. There is no known treatment for many NTM infections, but fortunately, MAC infection is curable with 2 or 3 antimicrobials for at least 12 months, including macrolides (clarithromycin and azithromycin), ethambutol, and rifamycins (rifampin or rifabutin) [22]. The same molecules are also effective against MABC and Mycobacterium kansasii, two NTMs that can also lead to severe diseases in humans [39]. Accurately detecting these NTM diseases is critical to ensure the most appropriate treatment for patients and combat drug resistance. This is equally imperative in HICs and LMICs.

\section{Conclusion}

Several diagnostic tools have been recently developed for TB and NTM, but many still present significant limitations given the current epidemiological features of these two similar diseases that have different treatment regimens. This has important public health implications including drug resistance and long-term eradication of these diseases. Therefore, tools that can simultaneously and accurately diagnose TB and NTM disease are urgently needed. Such tools will contribute not only in prescribing the right drug regimens to patients in a timely manner, but also in preventing antibiotics resistances, which is becoming an important threat to public health programs.

\section{Funding information}

This work was supported by the National Institutes of Health (R03AI137674, U54-EB027049, D43TW010350 and D71TW010428) and Northwestern Catalyzer Fund.

\section{Conflict of interest}

There are no conflicts of interest

\section{References}

1. WHO (2017) Global Tuberculosis Report 2017.

2. Prevots DR, Marras TK (2015) Epidemiology of human pulmonary infection with nontuberculous mycobacteria: a review. Clin Chest Med 36: 13-34. [Crossref]
3. Al Houqani M, Jamieson F, Chedore P, Mehta M, May K, et al. (2011) Isolation prevalence of pulmonary nontuberculous mycobacteria in Ontario in 2007. Can Respir $J$ 18: 19-24. [Crossref]

4. Asiimwe BB, Bagyenzi GB, Ssengooba W, Mumbowa F, Mboowa G, et al. (2013) Species and genotypic diversity of non-tuberculous mycobacteria isolated from children investigated for pulmonary tuberculosis in rural Uganda. BMC Infect Dis 13 : 88. [Crossref]

5. Badoum G, Saleri N, Dembele MS, Ouedraogo M, Pinsi G, et al. (2011) Failing a re-treatment regimen does not predict MDR/XDR tuberculosis: is "blind" treatment dangerous? Eur Respir J 37: 1283-1285. [Crossref]

6. Cassidy PM, Hedberg K, Saulson A, McNelly E, Winthrop KL (2009) Nontuberculous mycobacterial disease prevalence and risk factors: a changing epidemiology. Clin Infect Dis 49: e124-129. [Crossref]

7. Maiga M, Siddiqui S, Diallo S, Diarra B, Traore B, et al. (2012) Failure to recognize nontuberculous mycobacteria leads to misdiagnosis of chronic pulmonary tuberculosis. PLoS One 7: e36902. [Crossref]

8. Marras TK, Chedore P, Ying AM, Jamieson F (2007) Isolation prevalence of pulmonary non-tuberculous mycobacteria in Ontario, 1997 2003. Thorax 62: 661-666. [Crossref]

9. Marras TK, Mendelson D, Marchand-Austin A, May K, Jamieson FB (2013) Pulmonary nontuberculous mycobacterial disease, Ontario, Canada, 1998-2010. Emerg Infect Dis 19: 1889-1891. [Crossref]

10. Nyamogoba HDN, Mbuthia G, Mining S, Kikuvi G, Kikuvi R, et al. (2013) HIV co-infection with tuberculous and non-tuberculous mycobacteria in western Kenya: challenges in the diagnosis and management. African Health Sciences 12: 305-311. [Crossref]

11. Winthrop KL, McNelley E, Kendall B, Marshall-Olson A, Morris C, et al. (2010) Pulmonary nontuberculous mycobacterial disease prevalence and clinical features: an emerging public health disease. Am J Respir Crit Care Med 182: 977-982. [Crossref]

12. Maiga M, Abaza A, Bishai WR (2012) Current tuberculosis diagnostic tools \& role of urease breath test. Indian J Med Res 135: 731-736. [Crossref]

13. Hatta M, Sultan AR, Tandirogang N, Masjudi, Yadi (2010) Detection and identification of mycobacteria in sputum from suspected tuberculosis patients. BMC Res Notes 3: 72. [Crossref]

14. Reed JL, Basu D, Butzler MA, McFall SM (2017) XtracTB Assay, a Mycobacterium tuberculosis molecular screening test with sensitivity approaching culture. Sci Rep 7: 3653. [Crossref]

15. Chiang SS, Swanson DS, Starke JR (2015) New Diagnostics for Childhood Tuberculosis. Infect Dis Clin North Am 29: 477-502. [Crossref]

16. Nishiuchi Y, Iwamoto T, Maruyama F (2017) Infection Sources of a Common Nontuberculous Mycobacterial Pathogen, Mycobacterium avium Complex. Front Med (Lausanne) 4: 27. [Crossref]

17. Adjemian J, Olivier KN, Seitz AE, Holland SM, Prevots DR (2012) Prevalence of nontuberculous mycobacterial lung disease in U.S. Medicare beneficiaries. Am J Respir Crit Care Med 185: 881-886. [Crossref]

18. Akanbi MO, Achenbach C, Taiwo B, Idoko J, Ani A, et al. (2017) Evaluation of gene xpert for routine diagnosis of HIV-associated tuberculosis in Nigeria: A prospective cohort study. BMC Pulm Med 17: 87. [Crossref]

19. Bassirou DYT, Bakary K, Moumine S, Antieme CGT, Fatimata C, et al. (2017) Case Report: Extensively Drug Resistant Tuberculosis in Mali: lessons to be learned. BMC Res Notes 10: 51. [Crossref]

20. Zida S TZ, Kaboré A, Zingué D, Hien H, Sanou A, et al. (2014) Etat des lieux des mycobactérioses atypiques au Burkina Faso: résultats d'une enquête régionale. Pan Afr Med J 17: 188. [Crossref]

21. Nyamogoba HD, Mbuthia G, Mining S, Kikuvi G, Biegon R, et al. (2012) HIV coinfection with tuberculous and non-tuberculous mycobacteria in western Kenya: challenges in the diagnosis and management. Afr Health Sci 12: 305-311. [Crossref]

22. Ryu YJ, Koh WJ, Daley CL (2016) Diagnosis and Treatment of Nontuberculous Mycobacterial Lung Disease: Clinicians' Perspectives. Tuberc Respir Dis (Seoul) 79: 74-84. [Crossref]

23. Koh WJ (2017) Nontuberculous Mycobacteria-Overview. Microbiol Spectr 5. [Crossref]

24. Desikan P (2013) Sputum smear microscopy in tuberculosis: is it still relevant? Indian J Med Res 137: 442-444. [Crossref] 
25. Ngabonziza JC, Ssengooba W, Mutua F, Torrea G, Dushime A, et al. (2016) Diagnostic performance of smear microscopy and incremental yield of Xpert in detection of pulmonary tuberculosis in Rwanda. BMC Infect Dis 16: 660. [Crossref]

26. Anonymous (2000) Diagnostic Standards and Classification of Tuberculosis in Adults and Children. This official statement of the American Thoracic Society and the Center for Disease Control and Prevention was adopted by the ATS Board of Directors, July 1999. This statement was endorsed by the Council of the Infectious Disease Society of America, September 1999. Am J Respir Crit Care Med 161:1376- 1395. [Crossref]

27. van Zyl-Smit RN, Binder A, Meldau R, Mishra H, Semple PL, et al. (2011) Comparison of quantitative techniques including Xpert MTB/RIF to evaluate mycobacterial burden. PLoS One 6: e28815. [Crossref]

28. van Ingen J (2015) Microbiological diagnosis of nontuberculous mycobacterial pulmonary disease. Clin Chest Med 36: 43-54. [Crossref]

29. Huh HJ, Kim SY, Jhun BW, Shin SJ, Koh WJ (2018) Recent advances in molecular diagnostics and understanding mechanisms of drug resistance in nontuberculous mycobacterial diseases. Infect Genet Evol pii: S1567-1348(18)30784-30786. [Crossref]

30. Herold CD, Fitzgerald RL, Herold DA (1996) Current techniques in mycobacterial detection and speciation. Crit Rev Clin Lab Sci 33: 83-138. [Crossref]

31. Miller N, Hernandez SG, Cleary TJ (1994) Evaluation of Gen-Probe Amplified Mycobacterium Tuberculosis Direct Test and PCR for direct detection of Mycobacterium tuberculosis in clinical specimens. J Clin Microbiol 32: 393-397. [Crossref]

32. Yang M, Huh HJ, Kwon HJ, Kim JY, Song DJ, et al. (2016) Comparative evaluation of the AdvanSure Mycobacteria GenoBlot assay and the GenoType Mycobacterium CM/ AS assay for the identification of non-tuberculous mycobacteria. $J$ Med Microbiol 65: 1422-1428. [Crossref]
33. Sarkola A, Makinen J, Marjamaki M, Marttila HJ, Viljanen MK, et al. (2004) Prospective evaluation of the GenoType assay for routine identification of mycobacteria. Eur J Clin Microbiol Infect Dis 23: 642-645. [Crossref]

34. Helb D, Jones M, Story E, Boehme C, Wallace E, et al. (2010) Rapid detection of Mycobacterium tuberculosis and rifampin resistance by use of on-demand, near-patient technology. J Clin Microbiol 48: 229-237. [Crossref]

35. Reed JL, Walker ZJ, Basu D, Allen V, Nicol MP, et al. (2016) Highly sensitive sequence specific qPCR detection of Mycobacterium tuberculosis complex in respiratory specimens. Tuberculosis (Edinb) 101: 114-124. [Crossref]

36. Dorman SE, Schumacher SG, Alland D, Nabeta P, Armstrong DT, et al. (2018) Xper MTB/RIF Ultra for detection of Mycobacterium tuberculosis and rifampicin resistance: a prospective multicentre diagnostic accuracy study. Lancet Infect Dis 18: 76-84. [Crossref]

37. Porvaznik I, Solovic I, Mokry J (2017) Non-Tuberculous Mycobacteria: Classification, Diagnostics, and Therapy. Adv Exp Med Biol 944: 19-25. [Crossref]

38. Atif M, Sulaiman SA, Shafie AA, Ali I, Hassali MA, Saleem F (2012) WHO guidelines for treatment of tuberculosis: the missing links. Int J Clin Pharm 34: 506-509. [Crossref]

39. Kwon YS, Koh WJ (2016) Diagnosis and Treatment of Nontuberculous Mycobacterial Lung Disease. J Korean Med Sci 31: 649-659. [Crossref]

40. Hoefsloot W, van Ingen J, Andrejak C, Angeby K, Bauriaud R, et al. (2013) The geographic diversity of nontuberculous mycobacteria isolated from pulmonary samples: an NTM-NET collaborative study. Eur Respir J 42: 1604-1613. [Crossref]

Copyright: $\odot 2018$ Sarro YDS. This is an open-access article distributed under the terms of the Creative Commons Attribution License, which permits unrestricted use, distribution, and reproduction in any medium, provided the original author and source are credited. 